\title{
Brain-based Correlations Between Psychological Factors and Functional Dyspepsia
}

\author{
Jiaofen Nan, ${ }^{1}$ Jixin Liu, ${ }^{1 *}$ Junya Mu, ${ }^{1}$ Wanghuan Dun, ${ }^{2}$ Ming Zhang, ${ }^{2}$ Qiyong Gong, ${ }^{3}$ Wei Qin, ${ }^{1}$ Jie Tian, ${ }^{1}$ Fanrong Liang ${ }^{4}$, and \\ Fang Zeng ${ }^{4 *}$ \\ ${ }^{1}$ School of Life Science and Technology, Xidian University, Xi' an, China; ' ${ }^{2}$ Department of Medical Imaging, First Affiliated Hospital of Xi' an \\ Jiaotong University, Xi' an, China; ${ }^{3}$ Department of Radiology, The Center for Medical Imaging, Huaxi MR Research Center (HMRRC), West China \\ Hospital of Sichuan University, Chengdu, Sichuan, China; and ${ }^{4}$ The 3rd Teaching Hospital, Chengdu University of Traditional Chinese Medicine, \\ Chengdu, China
}

\section{Background/Aims}

Increasing evidence shows involvement of psychological disorders in functional dyspepsia (FD), but how psychological factors exert their influences upon FD remains largely unclear. The purpose of the present study was to explore the brain-based correlations of psychological factors and FD.

\section{Methods}

Based on Fluorine-18-deoxyglucose positron emission tomography-computed tomography, the altered cerebral glycometabolism was investigated in 40 FD patients compared with 20 healthy controls during resting state using statistical parametric mapping software.

\section{Results}

FD patients exhibited increased glucose metabolism in multiple regions relative to controls $(P<0.001$, family-wise error corrected). After controlling for the dyspeptic symptoms, increased aberrations persisted within the insula, anterior cingulate cortex (ACC), middle cingulate cortex (MCC) and middle frontal cortex (midFC), which was related to anxiety and depression score. Interestingly, FD patients without anxiety/depression symptoms also showed increased glycometabolism within the insula, ACC, MCC and midFC. Moreover, FD patients with anxiety/depression symptoms exhibited more significant hypermetabolism within the above 4 sites compared with patients without anxiety/depression symptoms.

Received: August 27, 2014 Revised: October 16, 2014 Accepted: October 16, 2014

(c) This is an Open Access article distributed under the terms of the Creative Commons Attribution Non-Commercial License (http://creativecommons. org/licenses/by-nc/3.0) which permits unrestricted non-commercial use, distribution, and reproduction in any medium, provided the original work is properly cited.

*Correspondence: Jixin Liu, PhD

School of Life Science and Technology, Xidian University, Xi'an 710071, China

Tel: +86-29-81891070, Fax: +86-29-81891060, E-mail: liujixin2010@gmail.com

Fang Zeng, MD

The 3rd Teaching Hospital, Chengdu University of Traditional Chinese Medicine, Chengdu, Sichuan 610075, China

Tel: +86-28-87784606, Fax: +86-28-87784606, E-mail: 0001170@cdutcm.edu.cn

*Jixin Liu and Fang Zeng are equally responsible for this study.

Financial support: This work was supported by the Project for the National Key Basic Research and development Program (973) under Grant No. 2012CB518501, 2014CB543203 and 2011CB707700, the National Natural Science Foundation of China under Grant No. 81471737, $81471811,81471738,81473602,81227901,81271644,30930112,81101036,81371530,81101108,31200837$, and 81371530 , the project of preeminent youth fund of Si Chuan province No. 2012JQ053 and the Fundamental Research Funds for the Central Conflicts of interest: None. Universities and the Knowledge Innovation Program of the Chinese Academy of Sciences under Grant No. KGCX2-YW-129.

Author contributions: Jiaofen Nan, processing data, interpreting data, and writing manuscript; Jixin Liu, designing study, collecting data and writing manuscript; Junya Mu, Wanghuan D, and Ming Zhang, processing data; Qiyong Gong, Wei Qin, Jie Tian, and Fanrong Liang, designing data and collecting data; and Fang Zeng: designing study, collecting data, and processing data.

ORCID: Jixin Liu, http://orcid.org/0000-0002-4239-1201. 


\section{Conclusions}

Our results suggested that the altered cerebral glycometabolism may be in a vicious cycle of psychological vulnerabilities and increased gastrointestinal symptoms.

(J Neurogastroenterol Motil 2015;21:103-110)

\section{Key Words}

Cerebral cortex; Dyspepsia; Glucose

\section{Introduction}

Functional dyspepsia (FD), one of the most common functional gastrointestinal disorders (FGID), is defined by the upper gastrointestinal syndromes in the absence of associations with obvious organic abnormalities. ${ }^{1}$ The clinical symptoms of this disorder mainly include recurrent upper abdominal fullness, early satiety, nausea and upper abdominal pain, which represent the patient's condition. ${ }^{1}$ Aside from suffering cardinal dyspepsia symptoms, patients with FD usually experienced higher scores of psychological aspects and stressful situations. ${ }^{2-4}$ For instance, anxiety and depression scores were often displayed above the normal range. ${ }^{2,3}$ However, what role psychological factors play in symptom generation of $\mathrm{FD}$ patients has not been completely elucidated.

An earlier dominant theory stated that psychological disorders merely increased the health-care-seeking behavior and utilization of medical services in FGID patients, ${ }^{5}$ suggesting that psychosocial factors may distinguish those that seek medical help from those that do not. In recent years, increasing evidence showed that the psychological components were partly relevant to gastrointestinal functions and symptoms. ${ }^{6-9}$ Moreover, anxiety or depression at baseline, found in a longitudinal study, predicted the presence of gastrointestinal symptoms after a 12-year follow-up. ${ }^{9}$ These studies suggested that the importance of psychological elements in the FD patients who frequently consult a physician. Recently, the advances of imaging technologies have provided a foundation for the study of psychological factors in FD patients. ${ }^{10}$ The Leuven group used $\mathrm{H}_{2}{ }^{15} \mathrm{O}$-PET to investigate the relationship between regional activity and anxiety scores in FD patients. ${ }^{11}$ They found anxiety scores correlated with patients' brain activity in the pregenual anterior cingulate cortex (ACC)/middle cingulate cortex (MCC) and dorsal pons. ${ }^{11}$ In addition, our previous study adopted tract- based spatial statistics to identify the abnormal alterations of the brain white matter in FD patients, and found that the inclusion of anxiety/depression scores as covariates removed some abnormalities of the microstructure measures including fractional anisotropy, mean diffusivity, axial diffusivity and radial diffusivity. ${ }^{12}$ The results suggested that anxiety and depression could account for a part of the white matter changes in FD patients. ${ }^{12}$ The above studies provided important insight on the role of psychological factors in FD. However, whether the observed central alterations are just a consequence of abnormal bottom-top signaling from the gastrointestinal tract to the brain remains to be elucidated.

Van Qudenhove and his colleague ${ }^{13}$ provided a summary of epidemiological studies that showed a relationship between FD and psychological/psychiatric disorders. They proposed an integrated biopsychosocial model of FD, supporting the notion that psychological disorders may induce a failure of the sensory filtering process causing an increase in physiological sensory signals within homeostatic-interoceptive regions. ${ }^{13}$ The defective sensory filtering may impair the functions of the gastrointestinal tract, such as food intake and perception of visceral stimuli. ${ }^{13}$ This model provided potential evidence for the phenomena that anxiety/depression was significantly correlated with the severity of symptoms in FGID patients. ${ }^{14,15}$ It is also supportive of the possibility that psychological factors are involved in the development of FD via homeostatic-interoceptive regions. Hence, we hypothesized that psychological variables may be interconnected with the dyspeptic symptoms via neural signals.

To test our hypothesis, we examined the cerebral glycometabolism of $40 \mathrm{FD}$ patients and 20 gender- and age-matched healthy subjects via fluorine-18-deoxyglucose positron emission tomography-computed tomography (PET-CT). First, we wanted to validate whether or not the altered central signals were merely a consequence of aberrant signaling from the upper abdomen to the brain in FD patients. Second, we aimed to test whether or not anxiety and depression were one of influencing factors on FD via neural signals. 


\section{Materials and Methods}

All subjects signed written informed consents before the study. This study protocol was approved by the Ethics Committee of Chengdu University of Traditional Chinese Medicine (No. 2011KL003).

\section{Subjects}

Forty FD patients and twenty healthy volunteers who served as controls were included in this study. Their ages ranged from 20 to 30 years (Table). All subjects underwent a series of physical and psychological assessments including a history of diseases and medication, gastrointestinal endoscopy, upper abdominal ultrasound and electrocardiogram, and an evaluation of mental health. None of the healthy controls showed gastrointestinal signs or a history of other serious disease. The patients were recruited according to Rome III criteria, ${ }^{1}$ and were excluded from the study if they met any one of the following criteria: (1) suffering from ulcers or erosive gastroduodenal lesions, gastric atrophy, cholecystitis or esophagitis; (2) having a history of drug abuse or trauma with loss of consciousness; (3) having experienced neurological or psychiatric disorders; (4) having respiratory, cardiovascular, renal or hepatic illness; (5) having other serious co-morbidities; and (6) being pregnant or lactating.

\section{Instruments}

Each patient completed a dyspepsia symptom questionnaire and a Nepean dyspepsia index. ${ }^{16,17}$ The major dyspeptic symptoms were assessed using a four item symptom questionnaire, which scores postprandial abdominal fullness or discomfort, early satiety, epigastric pain and burning on a four point scale. A higher score represents more severe dyspepsia complaints. The Nepean dyspepsia index evaluates the patient's quality of life in 25 dimensions on a five point scale. A higher score indicates better quality of life. Anxiety and depression were estimated using the Self-rating anxiety scale (SAS) ${ }^{18}$ and Self-rating depression scale (SDS). ${ }^{19}$

In the Chinese norm, SAS less than 50 and SDS less than 53 are considered to be in the normal range. ${ }^{20,21}$ To determine the effects of anxiety and depression, FD patients were divided into two subgroups ${ }^{20,21}$ : patients without anxiety/depression symptoms (SAS < 50 and SDS < 53) and patients with anxiety/depression symptoms (SAS $\geq 50$ or $\mathrm{SDS} \geq 53$ ), denoted by SAS/SDS (-) and SAS/SDS (+) separately.

\section{Positron Emission Tomography-Computed Tomography Data Acquisition}

The scan was performed on a Biograph Duo BGO scanner (Siemens, Munich, Germany) at the PET-CT center, Sichuan Provincial People's Hospital, Chengdu, China. Only one subject participated in the experiment per day. After the overnight fast, the subject checked his/her blood sugar level and blood pressure at $8 \mathrm{AM}$. Then, he/she was shown to a darkroom and sat there quietly for 20 minutes. Next, the participant was given an intravenous injection of fluorine-18 fluorodeoxyglucose (a tracer, synthesized with a Mini Tracer accelerator $0.11 \mathrm{MCi} / \mathrm{kg}$ dosage) on the back of the right hand. After a 40-minute rest for the uptake period, a PET-CT scan was performed with the subject's ears plugged, eyes blindfolded and head immobilized. During the scan, he/she was asked to neither fall asleep nor concentrate on a specific thing. The scan images were paralleled to a horizontal

Table. Demographic and Clinical Characteristics

\begin{tabular}{lcccccc}
\hline \multicolumn{1}{c}{ Items } & HC & SAS/SDS $(-)$ & SAS/SDS $(+)$ & $P$-value & $P$-value $^{\mathrm{b}}$ & $P$-value \\
\hline Gender (male/female) & $6 / 14$ & $5 / 17$ & $3 / 15$ & 0.40 & 0.60 & 0.35 \\
Age (yr) & $22.95 \pm 1.67$ & $22.05 \pm 1.94$ & $22.39 \pm 2.55$ & 0.19 & 0.11 & 0.42 \\
Weight (kg) & $53.6 \pm 7.74$ & $53.59 \pm 5.84$ & $53.06 \pm 6.24$ & 0.899 & 0.99 & 0.81 \\
Height (cm) & $165.2 \pm 7.15$ & $163.96 \pm 6.28$ & $162.17 \pm 7.28$ & 0.28 & 0.55 & 0.20 \\
Dyspepsia symptom scores & - & $3.77 \pm 1.27$ & $4.56 \pm 1.54$ & $<0.001$ & $<0.001$ & $<0.001$ \\
SAS & $35.56 \pm 6.31$ & $37.58 \pm 6.0$ & $52.99 \pm 3.35$ & $<0.001$ & 0.29 & $<0.001$ \\
SDS & $34.91 \pm 5.96$ & $36.61 \pm 5.80$ & $51.68 \pm 4.48$ & $<0.001$ & 0.36 & $<0.001$ \\
Quality of life (NDI) & $96.69 \pm 1.50$ & $79.57 \pm 8.30$ & $76.44 \pm 7.32$ & $<0.001$ & $<0.001$ & $<0.001$ \\
\hline
\end{tabular}

HC, healthy controls; FD, functional dyspepsia; SAS, Self-rating anxiety scale; SDS, Self-rating depression scale; SAS/SDS (-), FD patients without anxiety/depression symptoms (SAS $<50$ and SDS $<53$ ); SAS/SDS (+), FD patients with anxiety/depression symptoms (SAS $\geq 50$ or SDS $\geq 53$ ); NDI, Nepean dyspepsia index.

${ }^{\mathrm{a}} P$-value, FD patients vs. $\mathrm{HC} ;{ }^{\mathrm{b}} P$-value, SAS/SDS (-) vs. HC; ${ }^{\mathrm{c}} P$-value, SAS/SDS $(+)$ vs. HC.

Data are presented as mean $\pm \mathrm{SD}$. 
plane through the anterior commissure and posterior commissure line.

The data acquisition was implemented with the following parameters: 3-dimensional collection mode, $3 \mathrm{~mm}$ slice thickness, $1.5 \mathrm{~mm}$ slice interval, $256 \times 256$ matrix size and $3 \times 10^{9}$ total counts. The scan covered the whole brain and the collected data were reconstructed using ordered subset expectation maximization (6 iterations and 16 subsets).

\section{Data Preprocessing}

Data analysis was implemented using SPM8 (statistical parametric mapping software; The MathWorks, Inc, Natick, MA, USA; http:/www.fil.ion.ucl.ac.uk/spm/). The images for each participant were corrected for small movements, spatially normalizing to the Montreal Neurological Institute template space with a $2 \mathrm{~mm}$ isotropic resolution and smoothing with an isotropic Gaussian kernel of $6 \mathrm{~mm}$ full width at half maximum. The relative brain radioactivity (regional radioactivity/the global mean value) was used as an indicator of cerebral glucose metabolism.

\section{Statistical Methods}

The analysis of the clinical data was carried out using SPSS software (version 17.0, SPSS Inc, Chicago, IL, USA) and MATLAB (2010b, The Mathworks Inc).

To evaluate the brain changes, we performed the group-level contrasts of the image analysis by using a two-sample $t$ test $(P<$ 0.001, family-wise error [FWE] corrected) in SPM8. To distinguish the potential effect of dyspepsia from that of psychological factors on cerebral glucose metabolism, the dyspepsia symptoms and SAS/SDS used as covariates were controlled alternately using a linear regression model. The mean value of regional glycometabolism of each patient was extracted and correlated with the clinical variables using the Pearson's correlation coefficient.

\section{Results}

Clinical characteristics of healthy group and 2 subgroups of FD patients are shown in Table.

\section{Functional Dyspepsia Patients Versus Healthy Controls}

Compared with healthy controls, FD patients (Fig. 1A) displayed increased glycometabolism within medial frontal cortex (medFC), middle frontal cortex (midFC), superior frontal cortex (SFC), inferior frontal cortex, orbito-frontal cortex, ACC, MCC, posterior cingulate cortex, insula, amygdala, parahippocampal cortex, hippocampus, pallidus, putamen, caudate, hypothalamus, thalamus and somatosensory cortices, as shown in Supplementary Table. However, there was no significantly decreased brain glycometabolism in FD patients.

\section{Functional Dyspepsia Patients Versus Controls With a Covariate}

After controlling for the dyspeptic symptoms, group differences between FD patients and controls were removed or reduced within multiple regions (Fig. 1 and Supplementary Table), but they persisted within the bilateral midFC, bilateral medFC, bilateral SFC, bilateral ACC, bilateral insula, right MCC, left putamen and bilateral SI/SII (Fig. 1B and Supplementary Table). Moreover, SAS and SDS correlated markedly with the levels of glycometabolism within the remaining regions including the left insula, right ACC, right MCC and left midFC (Fig. 2).

After controlling for the SAS/SDS score, no significant
A
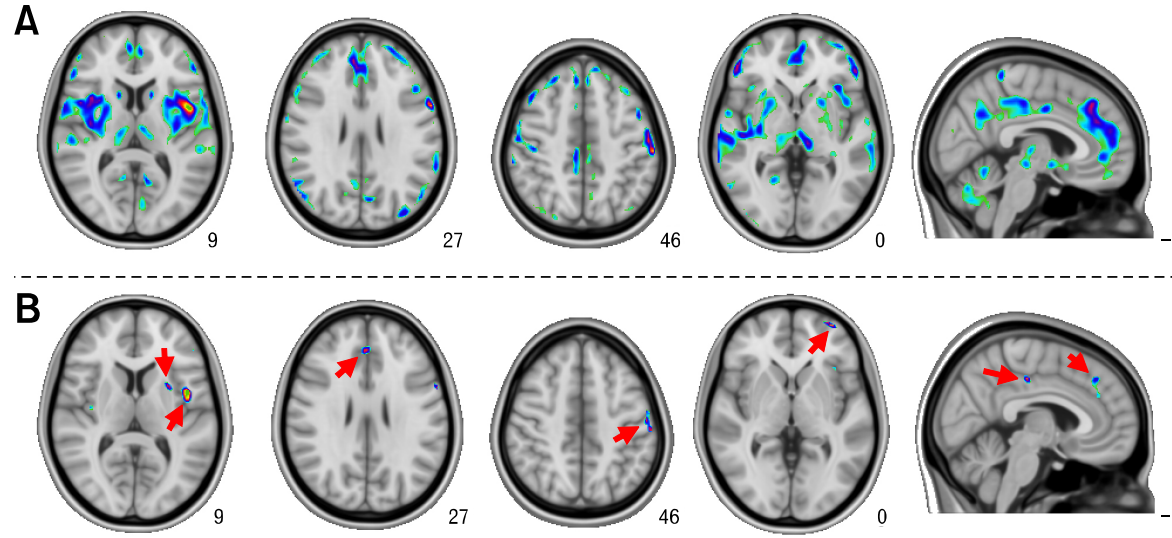

Figure 1. Glycometabolism differences between functional dyspepsia (FD) patients and healthy controls (HC). (A) FD $>\mathrm{HC}$ without covariates. (B) $\mathrm{FD}>$ $\mathrm{HC}$ with dyspeptic symptoms as covariates. 

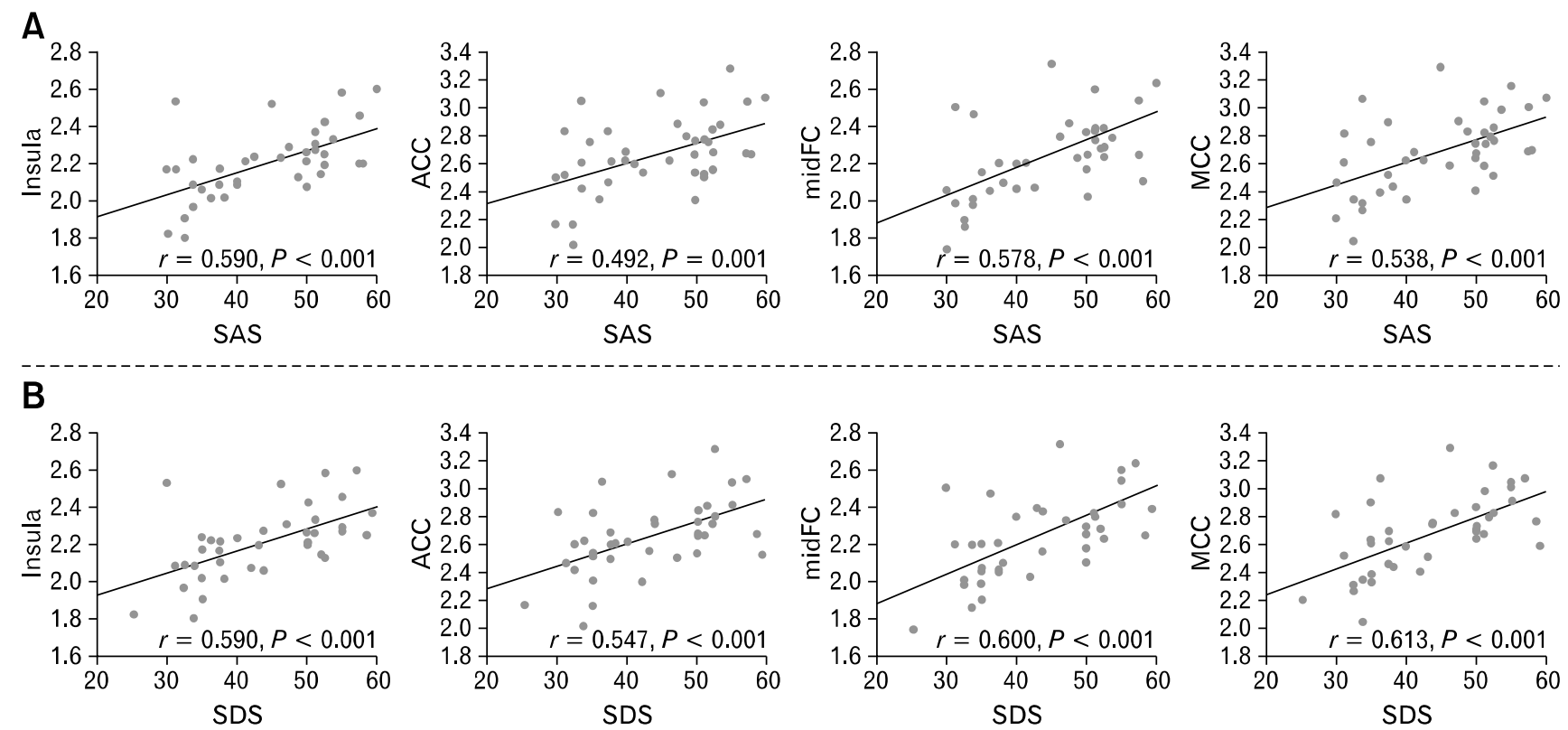

Figure 2. Correlation analysis of cerebral glycometabolism against the anxiety/depression score in functional dyspepsia patients. SAS, Self-rating anxiety scale; SDS, Self-rating depression scale; ACC, anterior cingulate cortex; MCC, middle cingulate cortex; midFC, middle frontal cortex.

A
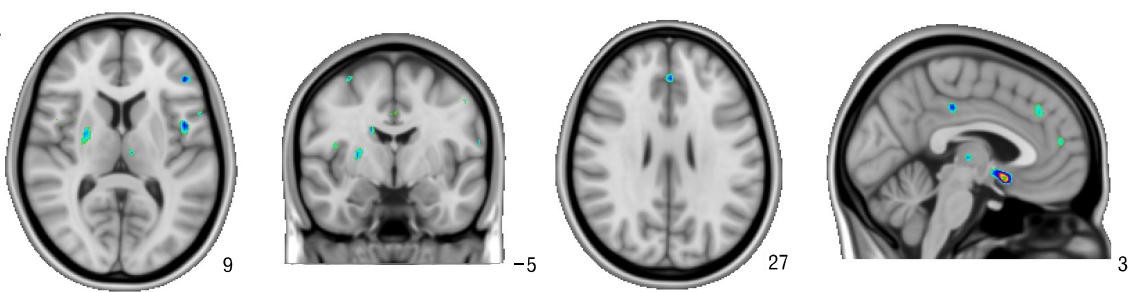

B
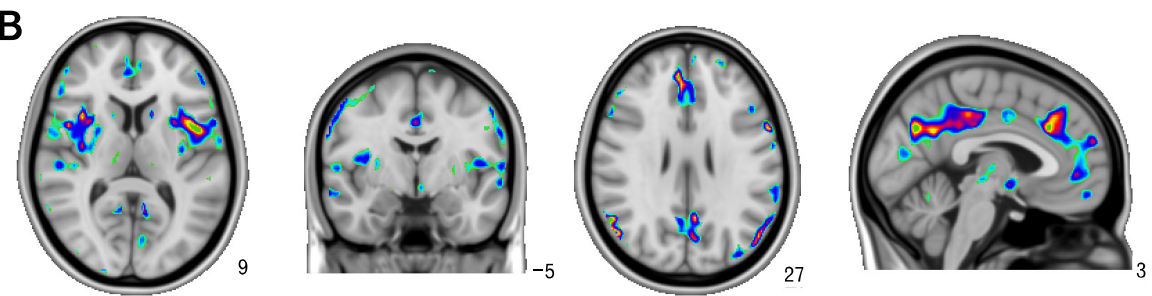

Figure 3. Increased cerebral glycometabolism in the two subgroups of functional dyspepsia (FD) patients relative to healthy controls (HC). (A) SAS/SDS $(-)>$ HC. (B) SAS/SDS $(+)>$ HC. SAS/SDS (-), FD patients without anxiety/depression symptoms; SAS/SDS $(+)$, FD patients with anxiety/depression symptoms.

group difference was found at $P<0.001$ after correction with FWE.

\section{Both Subgroups of Functional Dyspepsia Patients Versus Controls}

In contrast to controls, SAS/SDS (-) revealed significantly increased cerebral glycometabolism within multiple regions including the left insula, right ACC, right MCC and left midFC (Fig. 3A). Interestingly, the metabolic differences in the insula, $\mathrm{ACC}, \mathrm{MCC}$ and midFC disappeared when controlling for dys- peptic symptoms and persisted after controlling for anxiety and depression scores. The results corresponded to the clinical features: SAS/SDS (-) group and healthy group showed no significant difference in SAS and SDS (Table).

As expected, the SAS/SDS (+) group exhibited wider changes in glycometabolism relative to the healthy group, as opposed to the SAS/SDS ( - ) group (Fig. 3B). However, the metabolic differences between the SAS/SDS $(+)$ group and healthy group in the insula, ACC, MCC and midFC disappeared regardless of anxiety/depression scores or the presence of dyspeptic 
symptoms as covariates.

Although the increase of cerebral glycometabolism relative to healthy controls seemed greater in SAS/SDS $(+)$ group than in $\mathrm{SAS} / \mathrm{SDS}(-)$ group, given the significance threshold $\left(\mathrm{P}_{\mathrm{FWE}-\text { corrected }}\right.$ $<0.001)$, the subgroups of FD patients showed no significant differences.

\section{Discussion}

Our study focused on the correlations of psychological factors and dyspeptic symptoms grounded on cerebral glycometabolism, aiming to investigate the effects of anxiety/depression at the central level in FD patients. The study might provide a better understanding about the psychological aspects of FD.

In the current study, the metabolic abnormalities in $\mathrm{FD}$ patients persisted in the insula, ACC, MCC and midFC after controlling for the dyspepsia symptoms. Moreover, a significant positive correlation was found between anxiety/depression and the glycometabolism within the four regions (Fig. 2). These results indicated that the brain glucose metabolism in the four clusters may be disrupted by anxiety/depression in FD patients. In previous studies, Osuch et $\mathrm{al}^{22}$ and Kennedy et $\mathrm{al}^{23}$ investigated the relationship between anxiety/depression and cerebral metabolism via PET with ${ }^{18} \mathrm{~F}$-fluorodeoxyglucose. They found that anxiety/depression scores may be related directly to the regional metabolism in the prefrontal cortex (PFC), pregenual ACC as well as both the anterior and posterior insula. ${ }^{22,23}$ The results indicated that the close relationship between anxiety/depression and hypermetabolism/hypometabolism in the insula, ACC and PFC, which supported our results. Therefore, our findings of the abnormal brain metabolism controlled for the dyspepsia symptom in FD patients suggested that the increase in glucose metabolism may be partly an adaptive response to dysfunctional psychology rather than just abnormal signaling from the viscera to the brain.

Accumulating studies identified the activated brain sites during visceral stimulation in healthy subjects, ${ }^{24,25}$ investigating the responsiveness of the brain to visceral afferent information. In these studies, the commonly reported regions were the insula, ACC/MCC, PFC, thalamus and SI/SII. These observed regions represented their involvement in ascending projections of visceral afferents. Moreover, Moisset et $\mathrm{al}^{24}$ investigated the anatomical connection between sites involved in visceral sensations. They found that the neural circuits of visceral perception involved the insula, ACC/MCC, PFC and somatosensory cortices. ${ }^{24}$ These studies suggested that the insula, $\mathrm{ACC}, \mathrm{MCC}$ and $\mathrm{PFC}$ may have an intimate relationship with the input from the gastrointestinal tract. In the present study, the metabolic differences between the SAS/SDS ( - ) and healthy group were also found in the insula, ACC, MCC and midFC regardless of anxiety and depression scores as covariates, but disappeared when controlled for dyspeptic symptoms. The results exhibited correlations between cerebral glycometabolism in the four regions and dyspeptic symptoms. According to previous reports, ${ }^{13,26}$ the dyspeptic symptoms represent abdominal signals consciously perceived in the form of pain and/or discomfort. Therefore, our results may suggest the importance of the insula, ACC, MCC and midFC in dyspepsia-related sensations.

In a previous study, Phillips et $\mathrm{al}^{27}$ showed significantly higher brain activation in the insula and ACC as well as significantly greater discomfort and anxiety during esophageal stimuli with fearful faces than with neutral faces, providing first evidence for the neural mechanism underlying the influence of emotional context on symptoms in functional disorders. Although our results may not directly verify the role of psychological factors in visceral sensation, we showed the overlapping regions between dyspepsia symptoms and anxiety/depression. Hence, the psychological factors may be interacted with dyspepsia symptoms with the aid of the brain. This was consistent with the report that the insula, ACC, MCC and PFC straddle the lateral and medial systems of homeostatic pathways, processing sensory-discriminative and affective-motivational aspects of the discomfort perception. ${ }^{28}$ Intriguingly, SAS/SDS (+) group in the current study showed wider metabolic changes in the insula, ACC, MCC and midFC compared to SAS/SDS (-). This result seems to show that the anxiety/depression phenomenon may motivate the brain functional pattern to be adjusted to a more metabolically expensive organization. We inferred that the hypermetabolism may signal the viscera to meet its changes. That is to say, subsequent exposure to psychological disturbances may trigger or exacerbate dyspeptic symptoms. As Van Oudenhove and Aziz ${ }^{13}$ described in a biopsychosocial model, psychological morbidity may cause an increase in physiological sensory signals, leading to abdominal symptoms we perceive consciously, which is consistent with our findings. Therefore, there may be a vicious circle of gastrointestinal disturbances and psychological symptoms. This circle may explain the overlapping brain regions in our results for processing visceral information and affective regulation.

In a longitudinal study, Jones et $\mathrm{al}^{29}$ validated a model that early life factors may be the initial drivers of mood disturbance and gastrointestinal symptoms via increased neuroticism. This 
model showed the maintenance of gastrointestinal symptoms relying on an ongoing interaction between mood disorders and abdominal symptoms. ${ }^{29}$ In the present study, we found the correlations between anxiety/depression and dyspeptic symptoms based on glycometabolism in accordance with the model proposed by Jones et al. ${ }^{29}$ The insula, ACC, MCC and PFC may be crucial for both visceral condition and emotional situation, thus their abnormalities may modify the emotional response and visceral state, promoting gastrointestinal disorders. That is to say, psychological aspects or gastrointestinal tracts, facing uncertain threat, may lead to the triggering and exacerbation of dyspepsia symptoms. Therefore, it is likely that psychological phenomenon and gastrointestinal dysfunction have interchanged cause and effect in FD patients. This may be why the directionality between psychological disorders and gastrointestinal symptoms is still not settled. ${ }^{9}$

Curiously, a previous study ${ }^{30}$ examined the brain differences between FD patients and healthy controls using PET-CT but did not find the potential effects of anxiety and depression on the cerebral glycometabolism in FD patients. This was different from the current results. Clinically, this discrepancy may result from the gender distribution or patients' condition including anxiety/depression symptoms and dyspeptic symptoms because of the consistent study design and SPM analysis. To clarify the reason, we evaluated possible correlations between gender and the disease in brain glycometabolism. Results showed no significant clusters from cerebral glycometabolism comparisons conducted on all subjects to determine gender by FD interactions. Therefore, the gender distribution may not contribute to this discrepancy. We speculated that the major reason may be that most FD patients were in the normal range of anxiety and depression in the previous study ${ }^{30}$ as SAS/SDS $(-)$, whose SAS and SDS scores were similar to healthy subjects. In the current study, however, nearly half of the patients showed mild anxiety and depression. Therefore, more work is needed to determine essential elements of the psychological effects on FD patients and the applicability of psychological treatments in diverse populations.

There are still some limitations in the present study. The information on anxiety, depression and dyspepsia symptoms was evaluated based on the self-rating questionnaires. This assessment was subjective, thus there could be some bias. Furthermore, there were many influencing factors for the complicated psychology, such as chronic stresses from finances, fear, physical or sexual abuse and family discord. Therefore, in the further study, the influence of psychological components on FD should be understood in a more comprehensive way.
The study investigated the central mechanisms underlying a link between psychological factors and dyspeptic symptoms in FD patients. Results suggested that psychological factors may not just be an effect of FD; they may react on dyspepsia symptoms based on the brain-gut interaction. These findings may enhance our understanding of the interactions between psychological disturbances and gastrointestinal symptoms.

\section{Supplementary Materials}

Note: To access the supplementary table mentioned in this article, visit the online version of Journal of Neurogastroenterology and Motility at http://www.jnmjournal.org/, and at doi: 10.5056/jnm14096.

\section{References}

1. Drossman DA, Dumitrascu DL. Rome III: new standard for functional gastrointestinal disorders. J Gastrointest Liver Dis 2006;15: 237-241.

2. Tominaga K, Higuchi K, Iketani T, et al. Comparison of gastrointestinal symptoms and psychological factors of functional dyspepsia to peptic ulcer or panic disorder patients. Inflammopharmacology 2007;15:84-89.

3. Nakao H, Konishi H, Mitsufuji S, et al. Comparison of clinical features and patient background in functional dyspepsia and peptic ulcer. Dig Dis Sci 2007;52:2152-2158.

4. Wu JC. Psychological co-morbidity in functional gastrointestinal disorders: epidemiology, mechanisms and management. J Neurogastroenterol Motil 2012;18:13-18.

5. Koloski NA, Talley NJ, Boyce PM. Predictors of health care seeking for irritable bowel syndrome and nonulcer dyspepsia: a critical review of the literature on symptom and psychosocial factors. Am J Gastroenterol 2001;96:1340-1349.

6. Coen SJ, Yágüez L, Aziz Q, et al. Negative mood affects brain processing of visceral sensation. Gastroenterology 2009;137:253-261, e1-e2.

7. Labus JS, Mayer EA, Chang L, Bolus R, Naliboff BD. The central role of gastrointestinal-specific anxiety in irritable bowel syndrome: further validation of the visceral sensitivity index. Psychosom Med 2007;69:89-98.

8. Hall GBC, Kamath MV, Collins S, et al. Heightened central affective response to visceral sensations of pain and discomfort in IBS. Neurogastroenterol Motil 2010;22:276-e280.

9. Koloski NA, Jones M, Kalantar J, Weltman M, Zaguirre J, Talley $\mathrm{NJ}$. The brain-gut pathway in functional gastrointestinal disorders is bidirectional: a 12-year prospective population-based study. Gut 2012;61:1284-1290.

10. Budavari AI, Olden KW. Psychosocial aspects of functional gastrointestinal disorders. Gastroenterol Clin North Am 2003;32:477-506.

11. Van Oudenhove L, Vandenberghe J, Dupont P, et al. Abnormal regional brain activity during rest and (anticipated) gastric distension 
in functional dyspepsia and the role of anxiety: a H215O-PET study. Am J Gastroenterol 2010;105:913-924.

12. Zhou G, Qin W, Zeng F, et al. White-matter microstructural changes in functional dyspepsia: a diffusion tensor imaging study. Am J Gastroenterol 2013;108:260-269.

13. Van Oudenhove L, Aziz Q. The role of psychosocial factors and psychiatric disorders in functional dyspepsia. Nat Rev Gastroenterol Hepatol 2013;10:158-167.

14. Van Oudenhove L, Vandenberghe J, Vos R, Holvoet L, Demyttenaere $\mathrm{K}$, Tack J. Risk factors for impaired health-related quality of life in functional dyspepsia. Aliment Pharmacol Ther 2011;33:261-274.

15. Clauwaert N, Jones MP, Holvoet L, et al. Associations between gastric sensorimotor function, depression, somatization, and symptom-based subgroups in functional gastroduodenal disorders: are all symptoms equal? Neurogastroenterol Motil 2012;24:1088-e565.

16. Talley NJ, Haque M, Wyeth JW, et al. Development of a new dyspepsia impact scale: the Nepean Dyspepsia Index. Aliment Pharmacol Ther 1999;13:225-236.

17. Tian XP, Li Y, Liang FR, et al. Translation and validation of the Nepean Dyspepsia Index for functional dyspepsia in China. World J Gastroenterol 2009;15:3173-3177.

18. Zung WW. A rating instrument for anxiety disorders. Psychosomatics 1971;12:371-379.

19. Zung WW, Richards CB, Short MJ. Self-rating depression scale in an outpatient clinic: further validation of the SDS. Arch Gen Psychiatry 1965;13:508-515.

20. Wu WY. Self-rating anxiety scale (SAS). Shanghai Arch Psychol 1990;2:44.

21. Chunfang W, Zehuan C, Qing X. Self-rating depression scale (SDS): an analysis on 1340 health subjects. Chin J Nerv Men Dis 1986;12:
267-268.

22. Osuch EA, Ketter TA, Kimbrell TA, et al. Regional cerebral metabolism associated with anxiety symptoms in affective disorder patients. Biol Psychiatry 2000;48:1020-1023.

23. Kennedy SH, Evans KR, Krüger S, et al. Changes in regional brain glucose metabolism measured with positron emission tomography after paroxetine treatment of major depression. Am J Psychiatry 2001;158:899-905

24. Moisset X, Bouhassira D, Denis D, Dominique G, Benoit C, Sabaté JM. Anatomical connections between brain areas activated during rectal distension in healthy volunteers: a visceral pain network. Eur J Pain 2010;14:142-148.

25. Tillisch K, Mayer EA, Labus JS. Quantitative meta-analysis identifies brain regions activated during rectal distension in irritable bowel syndrome. Gastroenterology 2011;140:91-100.

26. Mayer EA, Tillisch K. The brain-gut axis in abdominal pain syndromes. Annu Rev Med 2011;62:381-396.

27. Phillips ML, Gregory LJ, Cullen S, et al. The effect of negative emotional context on neural and behavioural responses to oesophageal stimulation. Brain 2003;126(Pt 3):669-684.

28. Van Oudenhove L, Demyttenaere K, Tack J, Aziz Q. Central nervous system involvement in functional gastrointestinal disorders. Best Pract Res Clin Gastroenterol 2004;18:663-680.

29. Jones MP, Van Oudenhove L, Koloski N, Tack J, Talley NJ. Early life factors initiate a 'vicious circle' of affective and gastrointestinal symptoms: a longitudinal study. United European Gastroenterol J 2013;1:394-402.

30. Zeng $\mathrm{F}$, Qin $\mathrm{W}$, Liang $\mathrm{F}$, et al. Abnormal resting brain activity in patients with functional dyspepsia is related to symptom severity. Gastroenterology 2011;141:499-506. 
Supplementary Table. Foci With Significantly Increased Cerebral Glycometabolism in Functional Dyspepsia Patients Compared With Healthy Controls During Resting State

\begin{tabular}{|c|c|c|c|c|c|c|c|c|c|}
\hline \multirow{2}{*}{\multicolumn{2}{|c|}{ Regions }} & & \multirow{2}{*}{$\begin{array}{l}\text { Talairach } \\
\text { coordinates }\end{array}$} & \multicolumn{3}{|c|}{ Without any covariates } & \multicolumn{3}{|c|}{ Dyspeptic symptoms as covariates } \\
\hline & & & & $t$ & $\mathrm{BA}$ & Cluster size & t & $\mathrm{BA}$ & Cluster size \\
\hline \multirow{10}{*}{$\begin{array}{l}\text { Prefrontal } \\
\text { cortex }\end{array}$} & medFC & $\mathrm{L}$ & $(-4,54,-16)$ & 10.93 & $6,8-11,25,32$ & 692 & 7.2 & 11 & 21 \\
\hline & & $\mathrm{R}$ & $(6,33,35)$ & 11.09 & $6,8-11,25$ & 572 & 7 & 9 & 14 \\
\hline & $\operatorname{midFC}$ & $\mathrm{L}$ & $(-34,18,47)$ & 10.28 & $8-11,46,47$ & 658 & 8.18 & $8-10$ & 22 \\
\hline & & $\mathrm{R}$ & $(28,28,47)$ & 10.52 & & 909 & 6.96 & 8 & 10 \\
\hline & $\mathrm{SFC}$ & $\mathrm{L}$ & $(-34,16,51)$ & 12.53 & $8-11$ & 435 & 8.82 & 8,10 & 34 \\
\hline & & $\mathrm{R}$ & $(24,28,50)$ & 11.49 & & 538 & 7.59 & & 20 \\
\hline & IFC & $\mathrm{L}$ & $(-48,33,-5)$ & 11.68 & $9,10,13,44-47$ & 425 & & & \\
\hline & & $\mathrm{R}$ & $(42,28,-13)$ & 10.31 & & 532 & & & \\
\hline & OFC & $\mathrm{L}$ & $(-4,44,-21)$ & 8.4 & 11 & 37 & & & \\
\hline & & $\mathrm{R}$ & $(8,50,-19)$ & 8.32 & & 23 & & & \\
\hline \multirow{24}{*}{$\begin{array}{l}\text { Limbic } \\
\text { system }\end{array}$} & $\mathrm{ACC}$ & $\mathrm{L}$ & $(-2,42,-5)$ & 10.29 & $10,24,25,32$ & 319 & 6.9 & 32 & 10 \\
\hline & & $\mathrm{R}$ & $(4,36,24)$ & 10.27 & & 399 & 7.28 & & 14 \\
\hline & $\mathrm{MCC}$ & $\mathrm{L}$ & $(-4,-27,40)$ & 11.95 & $23,24,31,32$ & 579 & & & \\
\hline & & $\mathrm{R}$ & $(8,-21,40)$ & 10.73 & & 686 & 8.1 & 31 & 21 \\
\hline & PCC & $\mathrm{L}$ & $(-6,-56,16)$ & 9.43 & $23,30,31$ & 76 & & & \\
\hline & & $\mathrm{R}$ & $(8,-57,23)$ & 9.12 & & 45 & & & \\
\hline & Insula & $\mathrm{L}$ & $(-44,2,5)$ & 14.95 & & 484 & 8.2 & & 51 \\
\hline & & $\mathrm{R}$ & $(40,-11,10)$ & 12.34 & & 563 & 7.18 & & 11 \\
\hline & Amygdala & $\mathrm{L}$ & $(-28,-1,-18)$ & 8.33 & & 87 & & & \\
\hline & & $\mathrm{R}$ & $(18,-6,-11)$ & 8.43 & & 56 & & & \\
\hline & $\mathrm{PH}$ & $\mathrm{L}$ & $(-16,-39,-5)$ & 9.43 & & 192 & & & \\
\hline & & $\mathrm{R}$ & $(34,-30,-22)$ & 9.28 & & 72 & & & \\
\hline & Hippocampus & $\mathrm{L}$ & $(-30-35,-5)$ & 9.43 & & 10 & & & \\
\hline & & $\mathrm{R}$ & $(32,-22,-14)$ & 7.46 & & 6 & & & \\
\hline & Pallidus & $\mathrm{L}$ & $(-26,-12,1)$ & 8.48 & & 223 & & & \\
\hline & & $\mathrm{R}$ & $(12,4,-5)$ & 9.62 & & 204 & & & \\
\hline & Putamen & $\mathrm{L}$ & $(-26,6,9)$ & 9.84 & & 633 & 7.63 & & 13 \\
\hline & & $\mathrm{R}$ & $(16,11,-9)$ & 9.32 & & 650 & & & \\
\hline & Caudate & $\mathrm{L}$ & $(-12,12,7)$ & 10.92 & & 155 & & & \\
\hline & & $\mathrm{R}$ & $(16,10,11)$ & 10.95 & & 127 & & & \\
\hline & Hypothalamus & $\mathrm{L}$ & $(-4,-2,-7)$ & 10.45 & & 7 & & & \\
\hline & & $\mathrm{R}$ & $(4,-2,-7)$ & 8.31 & & 7 & & & \\
\hline & Thalamus & $\mathrm{L}$ & $(-4,-17,3)$ & 10.13 & & 249 & & & \\
\hline & & $\mathrm{R}$ & $(16,-19,12)$ & 10.2 & & 492 & & & \\
\hline \multirow{4}{*}{$\begin{array}{l}\text { Sensory } \\
\text { cortex }\end{array}$} & SI & $\mathrm{L}$ & $(-57,-21,40)$ & 11.28 & $1,2,3$ & 355 & 8.12 & $1,2,3$ & 22 \\
\hline & & $\mathrm{R}$ & $(59,-29,38)$ & 10.19 & & 467 & 7.21 & & 12 \\
\hline & SII & $\mathrm{L}$ & $(-59,-44,22)$ & 10.92 & 40 & 202 & 7.07 & 40 & 25 \\
\hline & & $\mathrm{R}$ & $(57,-31,40)$ & 11.2 & & 213 & 7.42 & & 28 \\
\hline
\end{tabular}

medFC, medial frontal cortex; midFC, middle frontal cortex; SFC, superior frontal cortex; IFC, inferior frontal cortex; OFC, orbito-frontal cortex; ACC, anterior cingulate cortex; MCC, middle cingulate cortex; PCC, posterior cingulate cortex; PH, parahippocampus; SI, primary somatosensory cortex; SII, secondary somatosensory cortex. 